\title{
HPV-negative head and neck squamous cell carcinomas: how can we use the tumor protein signature?
}

\author{
"What we need now, more than ever, is further research \\ to help develop a platform in order to validate biomarkers \\ and treatments in a prospective fashion specifically for \\ HPV-negative squamous cell carcinoma of the head and neck."
}

Keywords: biomarkers $\bullet$ head and neck cancer $\bullet$ HPV negative $\bullet$ p16 negative

Over the last decade our knowledge of squamous cell carcinoma of the head and neck (SCCHN) has been greatly expanded by the identification of human papillomavirus (HPV) as the causative factor of a large proportion of oropharyngeal cancers. p16 positivity, a surrogate marker of HPV initiated SCCHN, has proven to be a powerful prognostic tool demonstrating a $58 \%$ reduction in the risk of death in those with stage III/IV oropharyngeal cancer [1]. Our excitement must be tempered by the knowledge that HPV-positive SCCHN still represents a minority of total head and neck cancer cases [2]. For the foreseeable future, HPV-negative SCCHN will require ongoing efforts to improve therapeutic efficacy. Given the significantly worse outcomes among patients with HPV-negative SCCHN, highlighted by 5-year survival for locally advanced disease of approximately $40 \%$ [3], the investigational treatment paradigm has focused on identifying targeted agents that may synergize with chemotherapy and radiation to improve cure rates.

Unlike HPV-positive malignancies, up to $80 \%$ of HPV-negative tumors harbor mutations in TP53 [4], a key tumor suppressor protein, which leads to accumulation of genetic alterations giving rise to unchecked cell division and resistance to apoptosis. Mutations abrogating this protein's function, so-called disruptive mutations, appear most strongly associated with worse overall survival [5].
This highlights the importance of integrating genomic and proteinomic data to delineate how specific mutations impact protein function.

The proliferation of improved sequencing techniques has allowed for robust investigation into the mutational profile of SCCHN. Whole exome sequencing has demonstrated that HPV-negative tumors have approximately twice the rate of mutations compared with HPV initiated disease [6,7]. We know TP53 mutation helps engender gene mutation and amplification, thereby providing us a wealth of targetable gene mutations, while also presumably increasing the likelihood of clones resistant to standard therapy. Unfortunately, the heterogeneic nature of this process makes it is doubtful that any one targeted agent will be the 'silver bullet' for HPV-negative SCCHN. Our challenge then, is to identify viable targets, develop an appropriate method to assess for their presence and then find the right time to deploy therapy against them.

This search led us to the EGF receptor (EGFR) pathway, a pathway that is strongly activated in SCCHN. Rigorous investigation identified a number of potential targets within the EGFR pathway and among downstream and complementary pathways with significant cross-talk including Ras-MAPK, PI3K-PTEN-AKT and phospholipase $\mathrm{C}$ [8]. The dysregulation of these targets are hypothesized to contribute

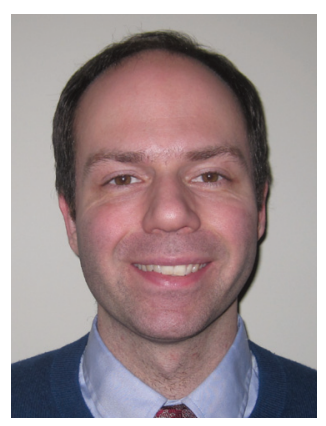

John Kaczmar

Fox Chase Cancer Center, Department of Medical Oncology, 333 Cottman Ave, Philadelphia, PA 19111, USA

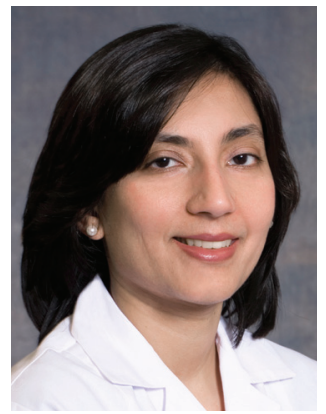

Ranee Mehra

Author for correspondence:

Fox Chase Cancer Center, Department of Medical Oncology, 333 Cottman Ave, Philadelphia, PA 19111, USA

Tel.: +1 2152144297

Fax: +1 2157283639

Ranee.Mehra@fccc.edu 
to both treatment resistance and the relatively poor outcomes of HPV-negative SCCHN. EGFR itself, gave rise to the first US FDA approved targeted agent in this disease, cetuximab. Unfortunately, cetuximab monotherapy has only provided a modest degree of benefit in the treatment of HPV-negative SCCHN [9].

There are many other important protein targets that both cross talk and substantially interact with the EGFR pathway, many of which have investigational therapeutics already under study, including: mTOR, MEK, SRC, MET, HDAC, aurora kinase, STAT, CCND1, CDKN2a, TGFB, SMAD4 and HIF-1 [8,1013]. Unrelated to this web of interaction, programmed death ligand-1, has garnered a great deal of excitement in the treatment of cancer, including SCCHN, as a potential marker for responsiveness to immunotherapy [14]. These myriad targets have helped shape the current research landscape in this disease entity thereby cultivating an ever-expanding ability to study a tumor's specific protein signature in an attempt to improve outcomes.

Despite the enthusiasm surrounding small molecule and antibody targeted therapies, standard treatments such as surgery or radiation for early-stage disease and the addition of sensitizing chemotherapy when more advanced or high-risk disease is present, will likely remain the cornerstones of therapy for some time. Our greater awareness of the molecular and proteinomic profile of SCCHN is important here as well and will provide further insight into the application of these standard therapies. For example, overexpression of ERCC1, a marker relevant to the nucleotide excision DNA repair pathway, and the KRAS variant have both been implicated in decreasing sensitivity to platinum agents and radiation [15-17]. So, in addition to guiding the development of brand new treatments, a greater understanding of tumor biology may better assist us in selecting the patients most likely to benefit from the our current standard of care.

\section{References}

1 Ang KK, Harris J, Wheeler R et al. Human papillomavirus and survival of patients with oropharyngeal cancer. N. Engl. J. Med. 363(1), 24-35 (2010).

2 Boscolo-Rizzo P, Del Mistro A, Bussu F et al. New insights into human papillomavirus-associated head and neck squamous cell carcinoma. Acta Otorhinolaryngol. Ital. 33(2), 77-87 (2013).

3 Ries Lag YJ, Keel GE, Eisner MP, Lin YD, Horner M-J. SEER survival monograph: cancer survival among adults: U.S. SEER Program, 1988-2001, patient and tumor characteristics. Natl Cancer Inst. SEER Progr. NIH Pub. No. 07-6215 (2007).
Targeted agents have been studied in combination with concurrent chemoradiation. Cetuximab (targeting EGFR), tirapazamine (targeting HIF-1 and hypoxia) and lapatanib (directed against EGFR and HER2/neu) have all been studied in conjunction with chemoradiation. While the addition of cetuximab to cisplatin and radiation failed to improve survival, there was a trend toward benefit of tirapazamine in HPV-negative SCCHN and a significant benefit of the addition of lapatanib, prompting continued study among HPV-negative patients [18-20]. However, it does not appear that specific protein signatures beyond p16 serve as relevant predictive biomarkers for these agents.

Thus overexpression of any one receptor or kinase is unlikely to reliably predict treatment response to a specific targeted agent. In the future, to optimally tailor therapy, we more than likely will utilize panels to identify gene mutations, protein expression and gene amplifications of each tumor to help guide selection of the optimal therapy. There are many promising targeted agents in the pipeline. What we need now, more than ever, is further research to help develop a platform in order to validate biomarkers and treatments in a prospective fashion specifically for HPV-negative SCCHN. Hopefully this will assist in selecting the appropriate agents to deploy from our ever-expanding therapeutic armamentarium to help further our ultimate goal - improving the survival of HPV-negative SCCHN.

\section{Financial \& competing interests disclosure}

$R$ Mehra has received consulting fees from Genentech, Novartis, Bayer and Bristol-Myers Squibb, and her spouse is an employee of GlaxoSmithKline. The authors have no other relevant affiliations or financial involvement with any organization or entity with a financial interest in or financial conflict with the subject matter or materials discussed in the manuscript apart from those disclosed.

No writing assistance was utilized in the production of this manuscript.

4 Leemans CR, Braakhuis BJ, Brakenhoff RH. The molecular biology of head and neck cancer. Nat. Rev. Cancer 11(1), 9-22 (2011).

5 Poeta ML, Manola J, Goldwasser MA et al. TP53 mutations and survival in squamous-cell carcinoma of the head and neck. N. Engl. J. Med. 357(25), 2552-2561 (2007).

6 Agrawal N, Frederick MJ, Pickering CR et al. Exome sequencing of head and neck squamous cell carcinoma reveals inactivating mutations in NOTCH1. Science 333(6046), 1154-1157 (2011).

7 Stransky N, Egloff AM, Tward AD et al. The mutational landscape of head and neck squamous cell carcinoma. Science 333(6046), 1157-1160 (2011). 
tness B, Bauman JE, Galloway T. Novel targets in HPV negative head and neck cancer: overcoming resistance to EGFR inhibition. Lancet Oncol. 14(8), e302-e309 (2013).

9 Vermorken JB, Herbst RS, Leon X, Amellal N, Baselga J. Overview of the efficacy of cetuximab in recurrent and/or metastatic squamous cell carcinoma of the head and neck in patients who previously failed platinum-based therapies. Cancer 112(12), 2710-2719 (2008).

10 Bussink J, Van Der Kogel AJ, Kaanders JH. Activation of the PI3-K/AKT pathway and implications for radioresistance mechanisms in head and neck cancer. Lancet Oncol. 9(3), 288-296 (2008).

11 Loyo M, Li RJ, Bettegowda C et al. Lessons learned from next-generation sequencing in head and neck cancer. Head Neck 35(3), 454-463 (2013).

12 Argiris A, Feinstein TM, Wang L et al. Phase I and pharmacokinetic study of dasatinib and cetuximab in patients with advanced solid malignancies. Invest. New Drugs. 30(4), 1575-1584 (2012).

13 Tian Q, Yuan J, Li Y, Jiang T, Gong H, Zhou W. Fast and automatic imaging of immunoenzyme-stained neuronal circuits in the whole brain of Drosophila. J. Biomed. Opt. 19(9), 90506 (2014).

14 Tanguy Y, Seiwert BB, Jared Weiss et al. A Phase Ib study of MK-3475 in patients with human papillomavirus (HPV)associated and non-HPV-associated head and neck (H/N) cancer. J. Clin. Oncol. 32(5; Suppl.), Abstract 6011 (2014).
15 Mehra R, Zhu F, Yang DH et al. Quantification of excision repair cross-complementing group 1 and survival in p16negative squamous cell head and neck cancers. Clin. Cancer Res. 19(23), 6633-6643 (2013).

16 Bauman JE, Austin MC, Schmidt R et al. ERCC1 is a prognostic biomarker in locally advanced head and neck cancer: results from a randomised, Phase II trial. Br. J. Cancer 109(8), 2096-2105 (2013).

17 Chung CH, Lee JW, Slebos RJ et al. A 3'-UTR KRASvariant is associated with cisplatin resistance in patients with recurrent and/or metastatic head and neck squamous cell carcinoma. Annal Oncol. 25(11), 2230-2236 (2014).

18 Ang KK, Zhang Q, Rosenthal DI et al. Randomized Phase III trial of concurrent accelerated radiation plus cisplatin with or without cetuximab for stage III to IV head and neck carcinoma: RTOG 0522. J. Clin. Oncol. 32(27), 2940-2950 (2014).

19 Harrington K, Berrier A, Robinson M et al. Randomised Phase II study of oral lapatinib combined with chemoradiotherapy in patients with advanced squamous cell carcinoma of the head and neck: rationale for future randomised trials in human papilloma virus-negative disease. Eur. J. Cancer 49(7), 1609-1618 (2013).

20 Rischin D, Young RJ, Fisher R et al. Prognostic significance of p16INK4A and human papillomavirus in patients with oropharyngeal cancer treated on TROG 02.02 Phase III trial. J. Clin. Oncol. 28(27), 4142-4148 (2010). 